\title{
The Intermediate Mass Black Hole Candidate in the Center of NGC 404: New Evidence from Radio Continuum Observations
}

\author{
Kristina Nyland ${ }^{1,2}$, \\ knyland@nmt.edu \\ Josh Marvil ${ }^{1,2}$, J. M. Wrobel ${ }^{2}$, Lisa M. Young ${ }^{1}$, B. Ashley Zauderer ${ }^{3}$ \\ Received —
}

To appear in an upcoming edition of ApJ

\footnotetext{
${ }^{1}$ New Mexico Tech, Department of Physics, 801 Leroy Place, Socorro, NM 87801

${ }^{2}$ National Radio Astronomy Observatory, Socorro, NM 87801, USA

${ }^{3}$ Harvard University, 60 Garden Street, Cambridge, MA 02138, USA
} 


\begin{abstract}
We present the results of deep, high-resolution, $5 \mathrm{GHz}$ Expanded Very Large Array (EVLA) observations of the nearby, dwarf lenticular galaxy and intermediate mass black hole candidate $\left(M_{\mathrm{BH}} \sim 4.5 \times 10^{5} \mathrm{M}_{\odot}\right)$, NGC 404 . For the first time, radio emission at frequencies above $1.4 \mathrm{GHz}$ has been detected in this galaxy. We found a modestly resolved source in the NGC 404 nucleus with a total radio luminosity of $7.6 \pm 0.7 \times 10^{17} \mathrm{~W} \mathrm{~Hz}^{-1}$ at $5 \mathrm{GHz}$ and a spectral index from 5 to $7.45 \mathrm{GHz}$ of $\alpha=-0.88 \pm 0.30$. NGC 404 is only the third central intermediate mass black hole candidate detected in the radio regime with subarcsecond resolution. The position of the radio source is consistent with the optical center of the galaxy and the location of a known, hard X-ray point source $\left(L_{\mathrm{X}} \sim 1.2 \times 10^{37} \mathrm{erg} \mathrm{s}^{-1}\right)$. The faint radio and X-ray emission could conceivably be produced by an X-ray binary, star formation, a supernova remnant or a low-luminosity AGN powered by an intermediate mass black hole. In light of our new EVLA observations, we find that the most likely scenario is an accreting intermediate mass black hole, with other explanations incompatible with the observed X-ray and/or radio luminosities or statistically unlikely.
\end{abstract}

Subject headings: galaxies: individual (NGC 404) — galaxies: nuclei — galaxies: active - galaxies: dwarf — galaxies: star formation — radio continuum: galaxies 


\section{Introduction}

It is widely accepted that supermassive black holes (SMBHs) with masses in the range $10^{6}-10^{9} \mathrm{M}_{\odot}$ are commonplace in galactic nuclei (Kormendy \& Richstone 1995). However, knowledge of the demographics and characteristics of black holes (BHs) in the centers of less massive galaxies is currently lacking. It is possible that many lower-mass galaxies simply host scaled-down versions of the central SMBHs in more massive galaxies, with masses $<10^{6} \mathrm{M}_{\odot}$ (McKernan et al. 2011). Such low BH masses would occupy the intermediate mass black hole (IMBH) regime (van der Marel 2004), which comprises the population of BHs with masses between those of stellar-mass BHs and SMBHs $\left(10^{2}-10^{6} \mathrm{M}_{\odot}\right)$. The detection and study of IMBHs promises to address a number of key astrophysical issues. For example, detections of IMBHs in low-mass galaxies would provide an excellent diagnostic tool for probing the formation mechanisms of 'seed' BHs (Volonteri 2010; van Wassenhove et al. 2010) as well as more exotic phenomena, such as gravity waves (Hughes 2009). Although there are a number of promising IMBH candidates in the centers of external galaxies, these detections have remained scarce, challenging and controversial (Greene \& Ho 2007b; Desroches et al. 2009; Barth et al. 2008). As a complication, it has been suggested that galaxies with total masses $<10^{10} \mathrm{M}_{\odot}$ may be dominated by massive nuclear star clusters (NSCs) rather than scaled-down versions of SMBHs (Ferrarese et al. 2006). There does exist a significant population of galaxies which appear to contain both a central massive BH and an NSC (Graham \& Spitler 2009; Seth et al. 2008). The fraction of galaxies harboring both an NSC and a central IMBH candidate is currently unknown, but a number of studies in recent years have explored such objects. Examples include some globular clusters which may actually be the stripped-down nuclei of former dwarf galaxies, such as $\omega$ Cen in the Galaxy (Noyola et al. 2010; van der Marel \& Anderson 2010), G1 in M31 (Gebhardt et al. 2002, 2005; Ulvestad et al. 2007; Kong et al. 2010), M54 in the Sagittarius dwarf galaxy (Ibata et al. 2009; Wrobel et al. 2011) and ESO 243-49 HLX-1 (Farrell et al. 
2012), though these cases have been controversial. Other well-known examples include the galaxies NGC 4395 (Filippenko \& Ho 2003; Peterson et al. 2005), Pox 52 (Barth et al.|2004; Thornton et al. 2008), Henize 2-101 (Reines et al.|2011), NGC 1042 (Shields et al. 2008), NGC 3621 (Barth et al. 2009), NGC 4178 (Satvapal et al. 2009), NGC 3367 and NGC 4536 (McAlpine et al. 2011) and NGC 404 (Seth et al. 2010; Binder et al. 2011).

NGC 404 is a nearby $(\mathrm{D}=3.1 \mathrm{Mpc})$, dwarf S0 galaxy with a complex nuclear environment. Unlike typical lenticular galaxies, which tend to be gas depleted with little signs of star formation (SF) and populate the red sequence on the color magnitude diagram (Baldry et al. 2004), NGC 404 appears to have been rejuvenated with cold gas during a merger 1 Gyr ago (del Río et al. 2004; Bouchard et al. 2010) and is currently undergoing low-level SF in a faint outer disk (Thilker et al. 2010) at a radius of a few hundred arcseconds (a few kpc). SF may have also occurred recently in the NGC 404 nucleus, as indicated by the detection of an NSC with an effective radius of $0.7^{\prime \prime}(10 \mathrm{pc})$ and a mass of $1.1 \pm 0.2 \times 10^{7} \mathrm{M}_{\odot}($ Ravindranath et al. 2001; Seth et al. 2010). Within the confines of the NSC, NGC 404 may also harbor a central IMBH. Compelling evidence in support of such an IMBH includes high resolution HST and ground-based adaptive optics dynamical studies (Seth et al. 2010). Seth et al. (2010) reported dynamical BH mass estimates of $M_{\mathrm{BH}}<10^{5} \mathrm{M}_{\odot}\left(\right.$ stellar dynamical upper limit) and $M_{\mathrm{BH}}=4.5_{-2.0}^{+3.5} \times 10^{5} \mathrm{M}_{\odot}$ (gas dynamical estimate).

Observations of the NGC 404 nucleus at a variety of wavelengths have provided substantial evidence for the presence of low-level accretion onto the candidate IMBH.

\footnotetext{
${ }^{1}$ The relatively uncertain central $\mathrm{BH}$ mass estimate in this dwarf galaxy of a few $\times 10^{6}$ $\mathrm{M}_{\odot}$ is not technically in the IMBH regime, but the error bars on this mass estimate span over an order of magnitude. Thus, more robust $M_{\mathrm{BH}}$ measurements may ultimately establish Henize 2-10 as an IMBH candidate.
} 
Hydrogen recombination line ratios have revealed that NGC 404 harbors a type 2 LINER 2 (Ho et al. 1997). Although the presence of a low-luminosity AGN (LLAGN) in all type 2 LINERs is a controversial subject, many type 2 LINERs are known to harbor accreting, massive BHs (Ho 2008). The Chandra analysis of NGC 404 by Binder et al. (2011) revealed a central, variable, hard $(2-10 \mathrm{keV})$ X-ray point source with a luminosity of $1.2_{-0.4}^{+0.7} \times 10^{37} \mathrm{erg} \mathrm{s}^{-1}$ and a power-law spectrum $(\Gamma=1.88)$. Although the weak, hard X-ray emission could be generated by a single X-ray binary (XRB) or an LLAGN, Binder et al. (2011) argued that the X-ray spectral shape and luminosity were most consistent with other observed LLAGNs and inconsistent with XRBs. Data at other wavelengths has also provided evidence for the presence of an LLAGN. In 2004, Satyapal et al. reported that the nuclear mid-IR emission lines of NGC 404 were consistent with bona fide AGNs in their sample. Near-IR data from Gemini have revealed unresolved and potentially variable hot dust emission, which is characteristic of Seyfert nuclei (Seth et al. 2010). In the UV regime, clear, yet shallow absorption features have indicated the presence of $\mathrm{O}$ stars in the center of NGC 404, along with an additional continuum component attributable to either an LLAGN or a population of less massive stars (Maoz et al. 1998). UV variability observed over a 9 year period also supports the existence of an LLAGN (Maoz et al. 2005).

A more thorough analysis of the nuclear engine of NGC 404 may ultimately help improve our understanding of SMBH growth and the relationship between massive $\mathrm{BH}$ evolution and the evolution of the host galaxies in which they reside. High-resolution, deep, interferometric radio observations offer the ability to easily detect and pinpoint emission from weakly accreting, low-mass BHs (Maccarone 2004). Yet, besides NGC 404, only two other candidate IMBHs, NGC 4395 (Ho \& Ulvestad 2001; Wrobel et al. 2001; Wrobel \& Ho

\footnotetext{
${ }^{2} \mathrm{~A}$ LINER is defined as a "low-ionization nuclear emission-line region;" type 2 LINERs lack the characteristic broad permitted lines present in type 1 AGNs. See Ho 2008 for review.
} 
2006) and GH 10 (Greene et al. 2006; Wrobel et al. 2008), have subarcsecond-scale radio continuum detections. In this paper, we present an analysis of our recent Expanded Very Large Array (EVLA) detection of parsec-scale radio emission in the NGC 404 nucleus and the implications it has on the status of NGC 404 as an IMBH candidate. In Section 2 we describe our observations and data reduction procedure. We discuss the possible physical interpretations of our EVLA observations in Section 3 and summarize our work in Section 4 .

\section{Observations}

We observed NGC 404 with the NRAO 3 EVLA (Perley 2011) at L-band (1-2 GHz) and $C$-band (4-8 GHz). The $L$-band observations were carried out in the B-configuration on April 11 and 30, 2011 (project ID: 10C-145) over 2 hours with a single 1 GHz-wide baseband centered at $1.5 \mathrm{GHz}$. The WIDAR correlator was configured with 8 spectral windows, each of which contained 64 channels with a frequency resolution of $2 \mathrm{MHz}$. These observations were phase-referenced to the calibrator J0119+3210 every 6 minutes with a switching angle of $4^{\circ}$. The positional accuracy of our phase calibrator was $<0.002^{\prime \prime}$. The calibrator $3 \mathrm{C} 48$ was used to set the amplitude scale to an accuracy of $3 \%$ and calibrate the bandpass. Four out of the 27 antennas experienced severe hardware malfunctions and were removed from the dataset. $L$-band data from the remaining antennas were flagged and calibrated with the Astronomical Image Processing System (AIPS) using standard procedures. We manually inspected each baseline and flagged the data using the AIPS task SPFLG to excise the heavy radio frequency interference $(\mathrm{RFI})$ present in the $L$-band frequency range.

The $C$-band observations were carried out in the A-configuration on July 9, 2011

\footnotetext{
${ }^{3}$ The National Radio Astronomy Observatory is a facility of the National Science Foundation operated under cooperative agreement by Associated Universities, Inc.
} 
(project ID: TDEM0009) over 30 minutes with two $1 \mathrm{GHz}$-wide basebands centered at 5 and $7.45 \mathrm{GHz}$. The WIDAR correlator was configured with 16 spectral windows split across the two basebands, each of which contained 64 channels with a frequency resolution of $2 \mathrm{MHz}$. These observations were phase-referenced to the calibrator J0111+3906 every 5 minutes with a switching angle of $3^{\circ}$. The positional accuracy of our phase calibrator was $<0.002^{\prime \prime}$. The calibrator $3 \mathrm{C} 48$ was used to set the amplitude scale to an accuracy of $3 \%$ and calibrate the bandpass. We flagged, calibrated and imaged the $C$-band data with the Common Astronomy Software Applications package (CASA). The WIDAR correlator malfunctioned during our observations and caused large amplitude jumps at scan boundaries, affecting intermediate frequencies B and C. We were able to correct this problem by applying switched power during data import to CASA and re-calibrating the gain. RFI was minimal in our chosen basebands and only a few isolated frequencies which displayed bright, narrowband RFI required flagging. Three out of the twenty-seven antennas contained significantly corrupted data and were partially removed when necessary.

We imaged the $L$ and $C$-band data in CASA with the CLEAN task using the Cotton-Schwab algorithm in the Multi Frequency Synthesis mode and Briggs weighting. For the $C$-band observations we smoothed the upper $7.45 \mathrm{GHz}$ baseband to match the resolution of the lower $5 \mathrm{GHz}$ baseband using the CASA task IMSMOOTH. We used the JMFIT task in AIPS to fit a single elliptical Gaussian component to the emission to determine its flux density, extent and position. Observational parameters are summarized in Table 1 and radio images with contours are shown in Figure 1. The arcsecond ( $\sim 50$ pc-scale) resolution observations at $L$-band reveal slightly resolved emission (Table 1). The sub-arcsecond ( $\sim 6$ pc-scale) resolution observations at $C$-band reveal modestly resolved emission (Table 1) consistent with the location of a central, hard X-ray point source (Binder et al. 2011) and the optical position of the nucleus (Seth et al. 2010), as shown in Figure 2, The $5 \mathrm{GHz}$ emission is centered at $\alpha_{\mathrm{J} 2000}=01^{\mathrm{h}} 09^{\mathrm{m}} 27.00^{\mathrm{s}}$ and $\delta_{\mathrm{J} 2000}=+35^{\circ} 43^{\prime} 04.91^{\prime \prime}$ (with a 1-D 
positional uncertainty of $0.1^{\prime \prime}$ dominated by calibration strategies). From the integrated flux densities at 5 and $7.45 \mathrm{GHz}$, we obtain a $C$-band spectral index of $\alpha=-0.88 \pm 0.30$ (where $\left.\mathrm{S} \propto \nu^{\alpha}\right)$. We also used our $1.5 \mathrm{GHz}$ flux density to compute the three-point radio spectral index of $\alpha=-1.15 \pm 0.05$. However, we caution that this provides only an estimate of the spectral index between 1.5 and $7.5 \mathrm{GHz}$ since the $1.5 \mathrm{GHz}$ data was an order of magnitude lower in resolution and not observed simultaneously with the 5 and $7.45 \mathrm{GHz}$ data.

\section{Discussion}

While the parsec-scale radio emission in the NGC 404 nucleus may originate from low-level accretion onto a central IMBH, this is not the only possibility. The radio emission could also conceivably be produced by a single XRB, a young supernova remnant (SNR) or a recent bout of $\mathrm{SF}$. We utilize the ratio of the $5 \mathrm{GHz}$ radio luminosity to the hard $(2-10 \mathrm{keV})$ X-ray luminosity, $R_{\mathrm{X}}=\nu L_{\nu}(5 \mathrm{GHz}) / L_{\mathrm{X}}($ Terashima \& Wilson 2003), to help distinguish among the different possibilities for the origin of the radio and X-ray emission. For NGC 404, we find $\log R_{\mathrm{X}}=-2.5$. We discuss the possibility and limitation of this ratio being explained by an X-ray binary (§3.1), star formation (§3.2), a supernova remnant (§3.3) and our preferred interpretation of an accreting IMBH (§3.4).

\subsection{X-ray Binary}

Although the X-ray luminosity of the compact, central source in NGC 404 of $\sim 1.2 \times 10^{37} \mathrm{erg} \mathrm{s}^{-1}$ could be generated by a single XRB (Binder et al. 2011), such objects are known to be radio-faint. To our knowledge, radio emission from an XRB beyond the

Milky Way has yet to be detected with certainty (Muxlow et al. 2010). Even a bright radio flare from a galactic XRB such as Cyg X-3 (D = $7 \mathrm{Kpc})$, which recently flared to 20 Jy 

still nearly a factor of two less than the radio power of NGC 404 extrapolated to $15 \mathrm{GHz}$ conservatively using our steeper, three-point radio spectral index of $\alpha=-1.15$. Thus, the radio luminosity of $\mathrm{NGC} 404$ is too high to have been generated by a typical XRB, let alone an XRB undergoing an extreme flaring event. This characteristic radio faintness of XRBs also manifests itself in their radio/X-ray ratios, which tend to be much lower than the radio/X-ray ratio of NGC 404. For instance, the radio/X-ray ratios of a sample of 7 galactic XRBs of various types have $\log R_{\mathrm{X}}<-5.3$ (Merloni et al. 2003; Reines et al. 2011). Therefore, an XRB origin cannot explain both the observed radio and X-ray emission in the NGC 404 nucleus.

\subsection{Star Formation}

Evidence for recent nuclear SF in the center of NGC 404 includes the presence of the NSC; young, massive stars (Maoz et al. 2005) and diffuse, soft X-ray emission (Binder et al. 2011). Seth et al. (2010) estimated a current star formation rate (SFR) upper limit of $1.0 \times 10^{-3} \mathrm{M}_{\odot} \mathrm{yr}^{-1}$ based on extinction-corrected nuclear $\mathrm{H} \alpha$ measurements from a $1^{\prime \prime}$ (15 pc) slit on the $6.5 \mathrm{~m}$ MMT. We used the following relation to estimate the total (thermal + nonthermal) radio flux expected from the current SFR at 1.5 and 5 GHz (Murphy et al. 2011):

$$
\begin{aligned}
& \left(\frac{\mathrm{SFR}_{\nu}}{M_{\odot} \mathrm{yr}^{-1}}\right)=10^{-27}\left[2.18\left(\frac{T_{\mathrm{e}}}{10^{4} \mathrm{~K}}\right)^{0.45}\left(\frac{\nu}{\mathrm{GHz}}\right)^{-0.1}+15.1\left(\frac{\nu}{\mathrm{GHz}}\right)^{\alpha_{\mathrm{NT}}}\right]^{-1} \\
& \times\left(\frac{L_{\nu}}{\operatorname{erg~s}^{-1} \mathrm{~Hz}^{-1}}\right) \text {. }
\end{aligned}
$$

We evaluated Equation 1 with $T_{\mathrm{e}}=10^{4} \mathrm{~K}$ and $\alpha_{\mathrm{NT}}=-0.8$ (Murphy et al. 2011) and 
found upper limits on the 1.5 and $5 \mathrm{GHz}$ contributions to SF of $1.3 \times 10^{25} \mathrm{erg} \mathrm{s}^{-1} \mathrm{~Hz}^{-1}$ $\left(1.3 \times 10^{18} \mathrm{~W} \mathrm{~Hz}^{-1}\right)$ and $5.7 \times 10^{24} \mathrm{erg} \mathrm{s}^{-1} \mathrm{~Hz}^{-1}\left(5.7 \times 10^{17} \mathrm{~W} \mathrm{~Hz}^{-1}\right)$, respectively. These luminosities correspond to flux densities of $1.1 \mathrm{mJy}$ at $1.5 \mathrm{GHz}$ and $0.5 \mathrm{mJy}$ at $5 \mathrm{GHz}$. These estimates indicate that a SF origin for the observed radio emission is indeed plausible, contrary to the conclusion of Binder et al. (2011). However, we point out that the predicted upper limit on the $1.5 \mathrm{GHz}$ flux due to $\mathrm{SF}$ is over a factor of 2.5 lower than our measured $1.5 \mathrm{GHz}$ flux of $2.83 \mathrm{mJy}$. The predicted upper limit on the $5 \mathrm{GHz}$ flux due to $\mathrm{SF}$ is also lower than the $5 \mathrm{GHz}$ flux density measured at parsec-scale resolution. This suggests that an additional emission source, such as a weak LLAGN, would be needed to provide the missing flux density.

\subsection{Supernova Remnant}

A single, young supernova remnant (SNR) could also be responsible for the radio emission. For instance, if the galactic SNR Cas A were moved to the distance of NGC 404, it would have $S_{1.4 \mathrm{GHz}}=2.8 \mathrm{mJy}$ and $S_{5 \mathrm{GHz}}=1.2 \mathrm{mJy}$ (Baars et al. 1977), roughly similar to the observed radio flux densities in NGC 404. The spatial extent of a Cas A-like SNR in NGC 404 would also be similar to the observed $5 \mathrm{GHz}$ radio size. At an age of about 300 years, Cas A has a diameter of $\sim 5 \mathrm{pc}$, similar to the observed $\sim 8 \mathrm{pc}$ extent of the emission in NGC 404. The well-measured radio spectral index of Cas A of $\alpha=-0.77$ is also similar to the $C$-band spectral index of $\alpha=-0.88$ for NGC 404 . However, the radio/X-ray ratio of Cas $\mathrm{A}$ is only $\log R_{\mathrm{X}} \sim-1.7$ (Reines et al. 2011) since SNRs tend to be relatively weak in the hard X-ray regime.

An utraluminous SNR, such as J1228+441 in the nearby galaxy NGC 4449, is another potential culprit for the X-ray and radio emission. J1228+441 is located at a distance of $3.8 \mathrm{Mpc}$ (Annibali et al. 2008) and is $60-200$ years old (Lacey et al. 2007). Like typical 
young SNRs, J1228+441 is compact with a diameter upper limit of $0.028^{\prime \prime}=0.5$ pc. Over three decades of radio observations, the flux densities and radio spectral indices of this young SNR have varied, with $5 \mathrm{GHz}$ luminosities ranging from $2.2 \times 10^{26} \mathrm{erg} \mathrm{s}^{-1} \mathrm{~Hz}^{-1}$ to $6.9 \times 10^{25} \mathrm{erg} \mathrm{s}^{-1} \mathrm{~Hz}^{-1}$ and a radio spectral index ranging from -0.6 to -1.0 from 1973 to 2002 (Lacey et al. 2007). The ratio of the $5 \mathrm{GHz}$ luminosity measured in 2002 to the 2-10 keV luminosity measured by Chandra in 2001 of $\sim 1.7 \times 10^{38} \mathrm{erg} \mathrm{s}^{-1}$ (Summers et al. 2003) gives $\log R_{\mathrm{X}} \sim-2.7$ (Reines et al. 2011), which is very similar to our $\log R_{\mathrm{X}}$ measurement in NGC 404. Although J1228+441 and NGC 404 share similar radio/X-ray ratios, we point out that many properties of the ultraluminous SNR in question are the result of the highly dense ISM in the host galaxy, NGC 4449. Therefore, such an extreme type of SNR is not expected in a galaxy with no evidence of a dense ISM, such as NGC 404 (Kuno et al. 2002).

Although an SNR origin for the radio emission remains a possibility, it is not the most likely scenario given the low SFR in the NGC 404 nucleus. We used the following relation with the SFR upper limit of $1 \times 10^{-3} \mathrm{M}_{\odot} \mathrm{yr}^{-1}$ (Seth et al. 2010) to estimate the supernova rate, $\dot{N}_{S N}$ (Murphy et al. 2011):

$$
\left(\frac{\mathrm{SFR}}{M_{\odot} \mathrm{yr}^{-1}}\right)=86.3\left(\frac{\dot{N}_{\mathrm{SN}}}{\mathrm{yr}^{-1}}\right)
$$

and found $\dot{N}_{S N} \approx 1 \times 10^{-5} \mathrm{yr}^{-1}$. The Poisson distribution gives a probability of $\sim 1 \%$ that at least one supernova has occurred in the nuclear region of NGC 404 in the last 1000 years. Given the low probability for a recent supernova, we conclude that a single, young SNR is not the most plausible explanation for the observed radio emission. Future Very Long Baseline Interferometric (VLBI) imaging with milliarcsecond-scale resolution would be able to more definitively rule out an SNR origin for the radio emission. 


\subsection{Accreting Intermediate-Mass Black Hole}

An IMBH-driven LLAGN origin for the radio and X-ray emission is a tantalizing possibility. The $C$-band spectral index of NGC 404 of $\alpha=-0.88$ is consistent with the wide range of radio spectral indices observed for LLAGNs (Ho \& Ulvestad 2001). The $\log R_{\mathrm{X}}$ value of NGC 404 of -2.5 is formally radio-loud, where radio-loud is defined as $\log \mathrm{R}_{\mathrm{X}}>-4.5$ (Terashima \& Wilson 2003). This is consistent with the typically radio-loud nature of LINERs and LLAGNs in general (Terashima \& Wilson 2003; Ho 2008). The median $\log \mathrm{R}_{\mathrm{X}}$ for all LLAGN subclasses ranges from -2.8 to -3.8 , and the median value for type 2 LINERs specifically is -2.9 (Ho 2008). It has been shown that as radio-loudness increases in LLAGNs, the Eddington ratio decreases (Ho 2002; Terashima \& Wilson 2003; Maccarone 2004). The Eddington ratio for the IMBH candidate in NGC 404, assuming a mass of $4.5 \times 10^{5} \mathrm{M}_{\odot}$, is $L_{\mathrm{Bol}} / L_{\mathrm{Edd}} \sim 3 \times 10^{-6}$ (where $L_{\mathrm{X}}=L_{\mathrm{Bol}} / 16$, Ho 2008). Thus, the IMBH in the center of NGC 404 is radio-loud and accreting well below its Eddington limit, similar to other weak LLAGNs. A comparison of the radio and X-ray properties of NGC 404 with other prominent IMBH candidates is provided in Table 2.

If we assume that the NGC 404 nucleus indeed harbors a BH, we can obtain a BH mass estimate by utilizing the "Fundamental Plane of Black Hole Activity" (FPBHA) (Merloni et al. 2003; Falcke 2004; Kording et al. 2006), which relates 5 GHz radio luminosity, 2-10 keV X-ray luminosity and BH mass for all BHs ranging from stellar-mass to SMBHs. The FPBHA is defined by:

$$
\log \left(L_{\mathrm{R}}\right)=\xi_{\mathrm{RX}} \log \left(L_{\mathrm{X}}\right)+\xi_{\mathrm{RM}} \log (M)+b_{\mathrm{R}}
$$

where the units of $L_{\mathrm{X}}$ are $\operatorname{erg~\mathrm {s}^{-1}}$, the units of $M$ are $\mathrm{M}_{\odot}$ and $L_{\mathrm{R}}=\nu L_{\nu} \operatorname{erg~s}^{-1}$.

The values of the empirically-derived correlation coefficients depend on the sample selection (Kording et al. 2006) and can provide insights into the underlying accretion physics. 
The Merloni et al. (2003) correlation coefficients are $\xi_{\mathrm{RX}}=0.60_{-0.11}^{+0.11}, \xi_{\mathrm{RM}}=0.78_{-0.09}^{+0.11}$ and $b_{\mathrm{R}}=7.33_{-4.07}^{+4.05}$. These correlation coefficients, along with the measured radio and X-ray luminosities of the IMBH candidate in the center of NGC 404, give a $\mathrm{BH}$ mass estimate of $\log (M) \sim 6.4 \pm 1.1 \mathrm{M}_{\odot}\left(2.5_{-2.3}^{+29.1} \times 10^{6} \mathrm{M}_{\odot}\right)$. This $\mathrm{BH}$ mass estimate is consistent, within the wide range of uncertainties, with the dynamical BH mass estimate of $\sim 4.5 \times 10^{5} \mathrm{M}_{\odot}($ Seth et al. 2010) $)$ as well as the BH mass predicted by the $M_{\mathrm{BH}}-\sigma_{*}$ relation of a few $\times 10^{5} \mathrm{M}_{\odot}$ for $\sigma_{*} \sim 40 \mathrm{~km} \mathrm{~s}^{-1}$ (Gultekin et al. 2009; Seth et al. 2010).

\section{Summary}

We observed the candidate IMBH in the NGC 404 nucleus with the EVLA in the B-configuration at $L$-band $(1-2 \mathrm{GHz})$ and the A-configuration at $C$-Band $(4-8 \mathrm{GHz})$ and detected, for the first time, radio emission at a frequency higher than $1.4 \mathrm{GHz}$. The $L$-band emission is slightly resolved on arcsecond $(\sim 50 \mathrm{pc})$ scales at $1.5 \mathrm{GHz}$ and has a radio power of $3.3 \pm 0.2 \times 10^{18} \mathrm{~W} \mathrm{~Hz}^{-1}$. The $5 \mathrm{GHz}$ emission is modestly resolved on subarcsecond $(\sim 6 \mathrm{pc})$ scales with a radio power of $7.6 \pm 0.7 \times 10^{17} \mathrm{~W} \mathrm{~Hz}^{-1}$ and a $C$-band spectral index from 5 to $7.45 \mathrm{GHz}$ of $\alpha=-0.88 \pm 0.30$. NGC 404 is only the third IMBH candidate with detected subarcsecond-scale radio continuum emission. The radio luminosity, spectral index and radio/X-ray ratio in the NGC 404 nucleus are most consistent with an IMBH-powered LLAGN. The location of the radio emission is consistent with the position of a hard X-ray point source (Binder et al. 2011) and the optical center of the galaxy. An XRB origin is unlikely given NGC 404's high $5 \mathrm{GHz}$ luminosity and radio/X-ray ratio $\left(\log R_{\mathrm{X}}=-2.5\right)$. A single, young SNR comparable to Cas A in the NGC 404 nucleus could produce emission similar to the observed $5 \mathrm{GHz}$ emission, but it could not explain the observed hard X-ray emission. An ultraluminous SNR similar to J1228+441 could conceivably produce the observed radio and X-ray emission, however, given the low supernova rate and lack of 
evidence for a dense ISM, the probability of this scenario is low. A SF origin for the emission remains a plausible scenario, although the upper limit on the predicted $5 \mathrm{GHz}$ flux density due to SF is less than that observed. The upper limit on the SF contribution to the $1.5 \mathrm{GHz}$ emission was deficient by over a factor of 2.5 compared to our measured $1.5 \mathrm{GHz}$ flux, further suggesting that SF alone may not be able to produce all of the observed emission. In summary, alternative explanations for the observed radio and X-ray emission exist but are either inconsistent with the observed X-ray and/or radio luminosities or are statistically unlikely. Furthermore, our FPBHA (Merloni et al. 2003) BH mass estimate is compatible with the gas dynamical mass estimate (Seth et al. 2010) and $M_{\mathrm{BH}}-\sigma_{*}$ relation estimates (Gultekin et al. 2009; Seth et al. 2010). Thus, to date, the most likely scenario for the compact $5 \mathrm{GHz}$ radio and hard X-ray emission in the nucleus of NGC 404 is an IMBH accreting at low levels.

A future EVLA study with the dedicated goal of mapping the radio spectral index at a variety of frequencies would allow us to better model the underlying emission as well as monitor the source for radio variability. Milliarcsecond-scale resolution VLBI imaging of the NGC 404 nucleus would provide a robust measurement of the brightness temperature capable of definitively ruling out a compact starburst, and may also reveal morphological features such as jets characteristic of some LLAGNs (Nagar et al. 2005). With ongoing sensitivity improvements at the Very Long Baseline Array, such observations of NGC 404 may be possible in the near future. Continued studies of the nuclei of nearby, low-mass galaxies such as NGC 404 will ultimately help improve our understanding of the interplay between BHs and galaxy evolution and may even provide deeper insight into the long-debated issue of the relationship between AGN feedback and nuclear SF.

We thank the anonymous referee for helpful suggestions that have improved the clarity and strength of this work. We also thank Anil Seth for helpful comments and for providing 
us with coordinate frame-corrected Hubble Legacy Archive images. The National Radio Astronomy Observatory is a facility of the National Science Foundation operated under cooperative agreement by Associated Universities, Inc. This research was partially funded by the NRAO Graduate Summer Student Research Assistantship Program.

Facilities: NRAO. 


\section{REFERENCES}

Annibali, F., Aloisi, A., Mack, J., Tossi, M. van der Marel, R. P. Angeretti, L., Leitherer, C., Sirianni, M. 2008, ApJ, 135, 1900

Baars, J. W. M., Genzel, R., Pauliny-Toth, I. I. K, Witzel, A. 1977, A\&A, 61, 99

Baldry, I. K., Glazebrook, K., Brinkmann, J., Ivezić, Ž., Lupton, R. H., Nichol, R. C., Szalay, A. S. 2004, ApJ, 600, 681

Barth, A. J., Ho, Luis, C., Rutledge, R. E. \& Sargent, W. L. W. 2004, ApJ, 607, 90

Barth, Aaron J., Greene, Jenny E., Ho, Luis C. 2008, ApJ, 136, 1179

Barth, Aaron J., Strigari, Louis E., Bentz, Misty C., Greene, Jenny E., Ho, Luis C. 2009, ApJ, 690, 1031

Binder, B., Williams, B. F., Eracleus, M., Seth, A. C., Dalcanton, J. J., Skillman, E. D., Weisz, D. R., Anderson, S. F., Gaetz, T. J., \& Plucinsky, P. P. 2011, ApJ, 737, 77

Bouchard, A., Prugniel, P., Koleva, M. \& Sharina, M. 2010, A\&A, 513, 54

Corbel, S., Dubus, G., Tomsick, J. A., Szostek, A., Corbet, R. H. D., Miller-Jones, J. C. A., Richards, J. L., Pooley, G., Trushkin, S., Dubois, R., Hill, A. B., Kerr, M., Max-Moerbeck, W., Readhead, A. C. S., Bodaghee, A., Tudose, V., Parent, D., Wilms, J. and Pottschmidt, K. 2012, MNRAS, accepted

Dalcanton, J. J., Williams, B. F., Seth, A. C., Dolphin, A., Holtzman, J., Rosema, K., Skillman, E. D., Cole, A., Girardi, L., Gogarten, S. M., Karachentsev, I. D., Olsen, K., Weisz, D., Christensen, C., Freeman, K., Gilbert, K., Gallart, C., Harris, J., Hodge, P., de Jong, R. S., Karachentseva, V., Mateo, M., Stetson, P. B., Tavarez, M., Zaritsky, D., Governato, F., Quinn, T. 2009, ApJS, 183, 67 
del Río, M. S., Brinks, E., \& Cepa, J., 2004, ApJ, 128, 89

Desroches, Louis-Benoit, Greene, Jenny E., Ho, Luis C. 2009, ApJ, 698, 1515

Falcke, H., Kording, E. \& Markoff, S. 2004, A\&A, 414, 895

Farrell, S. A, Servillat, M., Pforr, J., Maccarone, T. J., Knigge, C., Godet, O., Maraston, C., Webb, N. A., Barret, D., Gosling, A. J., Belmont, R., Wiersema, K. 2012, ApJ, 747,13

Ferrarese, L., Côté, P., Dalla Bontà, E., Peng, E. W., Merritt, D., Jordán, A., Blakeslee, J. P., Haşegan, M., Mei, S., Piatek, S., Tonry, J. L, West, M. J. 2006, ApJ, 644, 21

Filippenko, Alexei V. \& Ho, Luis C. 2003, ApJ, 588, 13

Gebhardt, Karl, Rich, R. M, Ho, Luis C. 2002, ApJ, 578, 41

Gebhardt, Karl, Rich, R. M, Ho, Luis C. 2005, ApJ, 634, 1093

Graham, A. W. \& Spitler, L. R. 2009, ApJ, 397, 2148

Greene, J. E., Ho, L. C., \& Ulvestad, J. S. 2006, ApJ, 636, 56

Greene, J. E., Ho, L. C. 2004, ApJ, 610, 722

Greene, J. E., Ho, L. C. 2007a, ApJ, 656, 84

Greene, J. E., Ho, L. C. 2007b, ApJ, 670, 92

Gultekin, K., Cackett, E. M., Miller, J. M., Di Matteo, T., Markoff, S., \& Richstone, D. O. 2009, ApJ, 706, 404

Ho, L. C. 2002, ApJ, 564, 120

Ho, L. C. 2008, ARA\&A, 46, 475 
Ho, L. C., Filippenko, A. V., Sargent, W. L. W 1997, ApJS, 112, 315

Ho, L. C. \& Ulvestad, J. S. 2001, ApJS, 133, 77

Hughes, S. A. 2009, ARA\&A, 47, 107

Ibata, R., Bellazzini, M., Chapman, S. C., Dalessandro, E., Ferraro, F., Irwin, M., Lanzoni, B., Lewis, G. F., Mackey, A. D., Miocchi, P., Varghese, A. 2009, ApJ, 699, 169

Johnson, Kelsey E., Leitherer, Claus, Vacca, William D., Conti, Peter S. 2000, AJ, 120, 1273

Karachentsev, I. D., Sharina, M. E., Makarov, D. I., Dolphin, A. E., Grebel, E. K., Geisler, D., Guhathakurta, P., Hodge, P. W., Karachentseva, V. E., Sarajedini, A., Seitzer, P. 2002, A\&A, 389, 812

Kong, A. K. H., Heinke, C. O., Di Stefano, R., Cohn, H. N., Lugger, P. M., Barmby, P., Lewin, W. H. G., Primini, F. A. 2010, MNRAS, 407, 84

Kording, E., Falcke, H., Corbel, S. 2006, A\&A, 456, 439

Kormendy, J. \& Richstone, R. 1995, ARA\&A, 33, 581

Kuno, Nario, Nakai, Naomasa, Sorai, Kazuo, Nishiyama, Kohta, Vila-Vilaó, Baltasar 2002, PASJ, 54, 555

Lacey, C. K., Goss, W. M., Mizouni, L. K. 2007, ApJ, 133, 2156

Maccarone, T. 2004, MNRAS, 351, 1049

Maoz, D., Koratkar, A. P., Shields, J. C., Ho, L. C., Filippenko, A. V., \& Sternberg, A. 1998, AJ, 116, 55

Maoz, D., Nagar, N. M., Falcke, H. \& Wilson, A. S. 2005, AJ, 625, 699 
McAlpine, W., Satyapal, S., Gliozzi, M., Cheung, C. C., Sambruna, R. M. \& Eracleous, Michael 2011, ApJ, 728, 25

McKernan, B., Ford, K. E. S., Yaqoob, T., Winter, L. M. 2011, MNRAS, 413, 24

Merloni, A., Heinz, S. \& Di Matteo, T. 2003, MNRAS, 345, 1057

Moran, Edward C., Eracleous, Michael, Leighly, Karen M., Chartas, George, Filippenko, Alexei V., Ho, Luis C., Blanco, Philip R. 2005, AJ, 129, 2108

Murphy, E. J., Condon, J. J., Schinnerer, E., Kennicutt, R. C., Calzetti, D., Armus, L., Helou, G., Turner, J. L., Aniano, G. \& Beirão, P. 2011, ApJ, 737, 67

Muxlow, T. W. B., Beswick, R. J., Garrington, S. T., Pedlar, A., Fenech, D. M., Argo, M. K., van Eymeren, J., Ward, M., Zezas, A., Brunthaler, A. 2010, MNRAS, 404, 109

Nagar, N. M., Falcke, H., Wilson, A. S. 2005, A\&A, 435, 521

Noyola, Eva, Gebhardt, Karl, Kissler-Patig, Markus, Lützgendorf, Nora, Jalali, Behrang, de Zeeuw, P. Tim, Baumagardt, Holger 2010, ApJ, 719, 60

Perley, R. A., Chandler, C. J., Butler, B. J., Wrobel, J. M. 2011, ApJ, 739, 1

Peterson, Bradley M., Bentz, Misty C., Desroches, Louis-Benoit, Filippenko, Alexei V., Ho, Luis C., Kaspi, Shai, Laor, Ari, Maoz, Dan, Moran, Edward C., Pogge, Richard W. \& Quillen, Alice C. 2005, ApJ, 632, 799.

Ravindranath, S., Ho, L. C., Peng, C. Y., Filippenko, A. V. \& Sargent, W. L. W. 2001, ApJ, 122, 653

Reines, A. E., Sivakoff, G. R., Johnson, K. E. \& Brogan, C. L. 2011, Nature, 470, 66

Satyapal, S., Sambruna, R. M. \& Dudik, R. P. 2004, A\&A, 414, 825 
Satyapal, S., Böker, T., McAlpine, W., Gliozzi, M., Abel, N. P., Heckman, T. 2009, ApJ, 704,439

Seth, A., Agüeros, M., Lee, D. \& Basu-Zych, A. 2008, ApJ, 678, 116

Seth, A. C., Cappellari, M., Neumayer, N., Caldwell, N., Bastian, N., Olsen, K., Blum, R. D., Debattista, V. P., McDermid, R., Puzia, T., Stephens, A. 2010, ApJ, 714, 713

Shields, Joseph C., Walcher, Jakob, Böker, Torsten, Ho, Luis C., Rix, Hans-Walter \& van der Marel, Roeland P. 2008, ApJ, 682, 104

Summers, L. K., Stevens, I. R., Strickland, D. K., Heckman, T. M. 2003, MNRAS, 342, 690

Terashima, Y., Wilson, A. S. 2003, ApJ, 583, 145

Thilker, D. A., Bianchi, L., Schiminovich, D., Gil de Paz, A., Seibert, M., Madore, B. F., Wyder, T., Rich, R. M., Yi, S., Barlow, T., Conrow, T., Forster, K., Friedman, P., Martin, C., Morrissey, P., Neef, S. \& Small, T. 2010, ApJ, 714, 171

Thim, F., Hoessel, J. G., Saha, A., Claver, J., Dolphin, A., Tammann, G. A. 2004, AJ, 127, 2322

Thornton, C. E., Barth, A. J., Ho, L. C., Rutledge, R. E., Greene, J. E. 2008, ApJ, 686, 892

Tremaine, Scott, Gebhardt, Karl, Bender, Ralf, Bower, Gary, Dressler, Alan, Faber, S. M., Filippenko, Alexei V., Green, Richard, Grillmair, Carl, Ho, Luis C., Kormendy, John, Lauer, Tod R., Magorrian, John, Pinkney, Jason, Richstone, Douglas 2002, ApJ, 574, 740

Ulvestad, James S., Greene, Jenny E. \& Ho, Luis C. 2007, ApJ, 661, 151

van der Marel, R. P. 2004, in Coevolution of Black Holes and Galaxies, ed. L.C. Ho (Cambridge: Cambridge Univ. Press), 37 
van der Marel, Roeland, P. \& Anderson, Jay 2010, ApJ, 710, 1063

van Wassenhove, S., Volonteri, M., Walker, M. G., Gair, J. R. 2010, MNRAS, 408, 1139

Volonteri, M. 2010, ARA\&A, 18, 279

Wrobel, J. M., Fassnacht, C. D. \& Ho, L. C. 2001, ApJ, 553, 23

Wrobel, J. M. \& Ho, L. C. 2006, ApJ, 646, 95

Wrobel, J. M., Greene, J. E., Ho, L. C. \& Ulvestad, J. S. 2008, ApJ, 686, 838

Wrobel, J. M., Greene, J. E., Ho, L. C. 2011, AJ, 142, 113 

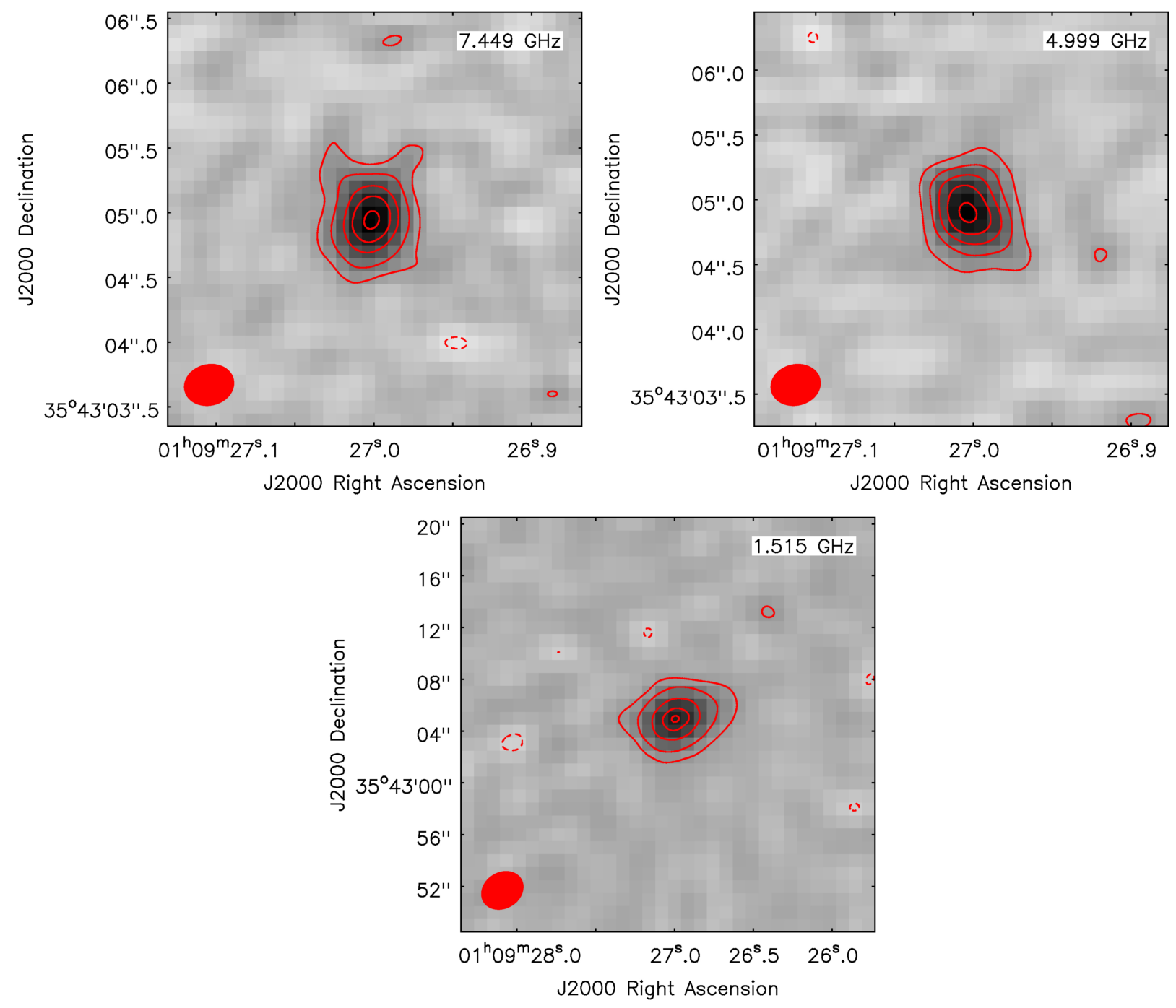

Fig. 1.- NGC 404 radio continuum images with contours at $7.45 \mathrm{GHz}, 5 \mathrm{GHz}$ and $1.5 \mathrm{GHz}$. The EVLA beam is the filled circle shown on the lower left in each image. For the $7.45 \mathrm{GHz}$ image, the relative contour levels are $[-3,3,6,10,13,16]$ and the unit contour level is $11 \mu \mathrm{Jy}_{\text {beam }}{ }^{-1}$. For the $5 \mathrm{GHz}$ image, the relative contour levels are $[-3,3,6,9,12,15]$ and the unit contour level is $16 \mu \mathrm{Jy}_{\text {beam }}{ }^{-1}$. For the $1.5 \mathrm{GHz}$ image, the relative contour levels are $[-3,3,8,20,32,39]$ and the unit contour level is $55 \mu \mathrm{Jy}_{\text {beam }}{ }^{-1}$. Note that $1^{\prime \prime}=15 \mathrm{pc}$. 


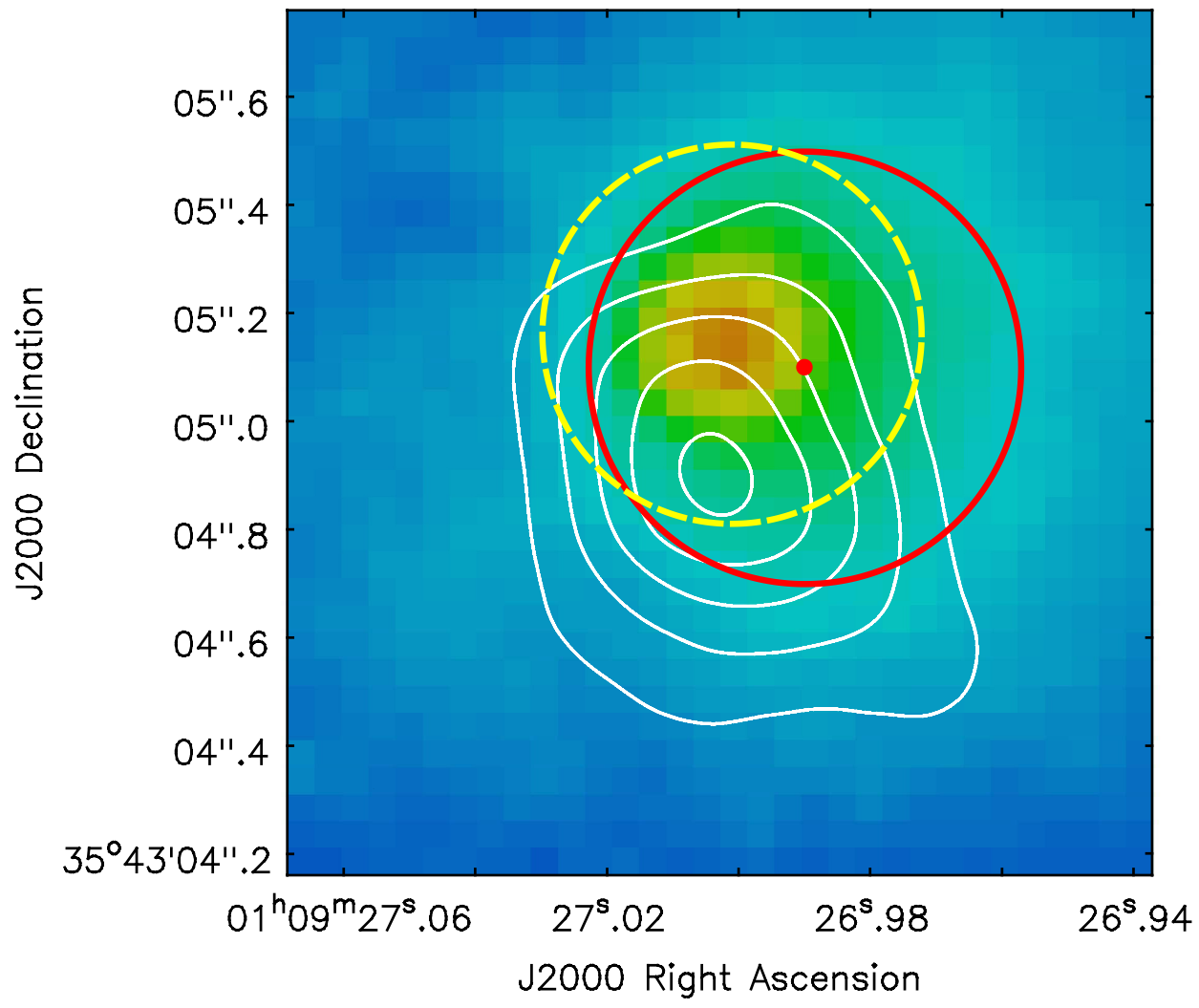

Fig. 2.- HST WFPC2 F814W image of the NGC 404 nucleus (Hubble Legacy Archive) with the EVLA $5 \mathrm{GHz}$ contours overlaid in white. The relative contour levels are $[-3,3,6,9,12,15]$ and the unit contour level is $16 \mu \mathrm{Jy}$ beam $^{-1}$. The red dot denotes the position of the hard X-ray point source (Binder et al. 2011) and the red circle illustrates the Chandra absolute positional uncertainty of $0.4^{\prime \prime}(6.0 \mathrm{pc})$. The dashed yellow circle shows the HST absolute positional uncertainty of $0.35^{\prime \prime}$ (5.3 pc) after registration with 2MASS point sources. The $5 \mathrm{GHz}$ source has a positional uncertainty of $0.1^{\prime \prime}(1.5 \mathrm{pc})$. Within the positional uncertainties, the $5 \mathrm{GHz}$ emission is consistent with the location of the hard X-ray point source and the optical center of NGC 404. 
Table 1. Summary of EVLA Observations of NGC 404

\begin{tabular}{|c|c|c|c|c|c|c|c|c|}
\hline \multirow[b]{2}{*}{$\begin{array}{l}\text { Frequency } \\
\qquad(\mathrm{GHz})\end{array}$} & \multirow[b]{2}{*}{$\begin{array}{c}\mathrm{rms} \\
\left(\mu \mathrm{Jy}_{\left.\text {beam }^{-1}\right)}\right.\end{array}$} & \multicolumn{2}{|c|}{ Beam Parameters } & \multicolumn{5}{|c|}{ Source Parameters } \\
\hline & & $\begin{array}{c}\theta_{M} \times \theta_{m} \\
\left({ }^{\prime \prime}\right)\end{array}$ & $\begin{array}{l}\text { P.A. } \\
(\operatorname{deg})\end{array}$ & $\begin{array}{c}\theta_{M} \times \theta_{m} \\
\left({ }^{\prime \prime}\right)\end{array}$ & $\begin{array}{l}\text { P.A. } \\
\text { (deg) }\end{array}$ & $\begin{array}{c}M \times m \\
(\mathrm{pc})\end{array}$ & $\begin{array}{l}\text { Total Flux Density } \\
\qquad(\mathrm{mJy})\end{array}$ & $\begin{array}{l}\log \left(P_{\text {rad }}\right) \\
\left(\mathrm{W} \mathrm{Hz}^{-1}\right)\end{array}$ \\
\hline$(1)$ & $(2)$ & $(3)$ & $(4)$ & $(5)$ & $(6)$ & $(7)$ & $(8)$ & (9) \\
\hline 7.45 & 12 & $0.39 \times 0.32$ & 103 & $0.54 \pm 0.05 \times 0.36 \pm 0.06$ & $19.7 \pm 10.0$ & $8.1 \pm 0.8 \times 5.4 \pm 0.9$ & $0.47 \pm 0.04$ & 17.73 \\
\hline 5 & 16 & $0.39 \times 0.32$ & 103 & $0.56 \pm 0.05 \times 0.35 \pm 0.05$ & $178.2 \pm 13.4$ & $8.4 \pm 0.8 \times 5.3 \pm 0.8$ & $0.66 \pm 0.06$ & 17.88 \\
\hline 1.5 & 55 & $3.42 \times 2.79$ & 122 & $2.32 \pm 0.22 \times 1.33 \pm 0.26$ & $101.5 \pm 10.7$ & $34.9 \pm 3.3 \times 20.0 \pm 3.9$ & $2.83 \pm 0.14$ & 18.51 \\
\hline
\end{tabular}

Note. - Col. (1): Center frequency. Col. (2): Average rms noise in image. Col. (3): Clean beam major $\times$ minor axis. Col. (4): Clean beam position angle. Col. (5): Angular dimensions (major $\times$ minor axis) of the deconvolved emission and error bars from JMFIT in AIPS. Col. (6): Position Angle of the deconvolved emission from JMFIT in AIPS. Col. (7): Linear dimensions (major $\times$ minor axis) of the deconvolved emission assuming a distance of 3.1 Mpc. Col. (8): Total integrated flux density and error. The error shown is the sum of the error reported by JMFIT in AIPS and the $3 \%$ calibration error, added in quadrature. Col. (9): Log of the radio power assuming a distance of $3.1 \mathrm{Mpc}$. 
Table 2. IMBH Candidates with High-Resolution Radio and X-ray Data

\begin{tabular}{|c|c|c|c|c|c|c|c|c|c|c|c|c|c|c|}
\hline Name & Morph. & $\begin{array}{c}\mathrm{D} \\
(\mathrm{Mpc})\end{array}$ & Ref. & $\begin{array}{c}\log \left(M_{\mathrm{BH}}\right) \\
\left(\mathrm{M}_{\odot}\right)\end{array}$ & Method & Ref. & $\begin{array}{c}\log \left(L_{\mathrm{R}}\right) \\
\left(\mathrm{erg} \mathrm{s}^{-1}\right)\end{array}$ & $\alpha$ & Ref. & $\begin{array}{c}\log \left(L_{\mathrm{X}}\right) \\
\left(\mathrm{erg} \mathrm{s}^{-1}\right)\end{array}$ & Ref. & $\log \left(R_{\mathrm{X}}\right)$ & $L_{\mathrm{Bol}} / L_{\mathrm{Edd}}$ & Ref. \\
\hline (1) & $(2)$ & $(3)$ & $(4)$ & (5) & $(6)$ & $(7)$ & $(8)$ & $(9)$ & $(10)$ & (11) & (12) & (13) & (14) & (15) \\
\hline NGC 404 & dSo & 3.1 & 1,2 & $5.65 \pm 0.25$ & I & 7 & 34.58 & -0.88 & 10 & 37.10 & 13 & -2.5 & -5.5 & 10 \\
\hline NGC 4395 & $\mathrm{Sdm}$ & 4.3 & 3 & $5.56 \pm 0.12$ & II & 8 & 34.95 & -0.60 & 11 & 39.91 & 14 & -5.0 & -2.9 & 8 \\
\hline GH 10 & $\cdots$ & 363.0 & 4,5 & $5.93 \pm 0.50^{\mathrm{b}}$ & III & 4 & 38.76 & -0.69 & 12 & 42.28 & 5 & -3.5 & 0.3 & 4 \\
\hline Henize $2-10^{\mathrm{a}}$ & $\mathrm{BCD}$ & 9.0 & 6 & $6.3 \pm 1.1$ & IV & 9 & 35.87 & -0.39 & 9 & 39.43 & 9 & -3.6 & -4.0 & 9 \\
\hline
\end{tabular}

${ }^{a}$ The central black hole mass estimate for Henize 2-10 is above $10^{6} \mathrm{M}_{\odot}$ and it is therefore not, strictly speaking, an IMBH candidate. However, the mass estimate is quite uncertain and the lower limit of the estimate lies within the IMBH range.

${ }^{\mathrm{b}}$ No uncertainty was given for the estimate of $M_{\mathrm{BH}}$ in GH 10 in Greene \& Ho (2004). However, the authors stated that their estimated black hole virial masses have a scatter of $\sim 0.5$ dex around the $M_{\mathrm{BH}}-\sigma_{*}$ relation, and we have adopted this value as a rough estimate of the uncertainty.

Note. - Col. (2): Galaxy morphology. Col. (3): Distance. Col. (4): Distance reference. Col. (5): Log of the black hole mass. Col. (6): Method of black hole mass estimation. I: dynamical measurement, II: reverberation mapping, III: H $\alpha$ line width-luminosity mass scaling relation and IV: Fundamental Plane of Black Hole Activity. Col. (7): Black hole mass reference. Col. (8): Log of the $5 \mathrm{GHz}$ radio luminosity (where $L_{\mathrm{R}}=\nu L_{\nu}(5 \mathrm{GHz})$ ). Col. (9): Radio spectral index, defined by $\mathrm{S} \propto \nu^{\alpha}$. For NGC 404 the radio spectral index is between 5 and $7.45 \mathrm{GHz}$, for NGC 4395 and GH 10 it's between 1.4 and 5 GHz and for Henize 2-10 it's between 5 and $8.5 \mathrm{GHz}$. Col. (10): Radio data reference. Col. (11): Log of the 2-10 keV X-ray luminosity. Col. (12): X-ray luminosity reference. Col. (13): Log of the radio/X-ray ratio, defined as $R_{\mathrm{X}}=\nu L_{\nu}(5 \mathrm{GHz}) / L_{\mathrm{X}}$. Col. (14): Log of the Eddington ratio. Col. (15): Eddington ratio reference.

References. - (1) Karachentsev et al. 2002; (2) Dalcanton et al. 2009; (3) Thim et al. 2004; (4) Greene \& Ho 2004; (5) Greene \& Ho 2007a (6) Johnson et al. 2000; (7) Seth et al. 2010; (8) Peterson et al. 2005; (9) Reines et al. 2011; (10) This work; (11) Ho et al. 2001; (12) Wrobel et al. 2008; (13) Binder et al. 2011; (14) Moran et al. 2005 